\title{
Atom-Probe Tomographic Study of Precipitation in an Ultrafine-grained Al-Zn-Mg- Cu Alloy (Al 7075)
}

\author{
Haiming Wen ${ }^{1,2}$, Kaka Ma ${ }^{1}$, Dieter Isheim ${ }^{2,3}$, David Seidman ${ }^{2,3}$, Julie M. Schoenung ${ }^{1}$, Enrique J. \\ Lavernia $^{1}$ \\ 1. Department of Chemical Engineering and Materials Science, University of California, Davis, CA, \\ 95616 \\ 2. Department of Materials Science and Engineering, Northwestern University, Evanston, IL 60208-3108 \\ 3. Northwestern University Center for Atom Probe Tomography (NUCAPT), Evanston, IL 60208-3108
}

Precipitation-hardened alloys can be further strengthened by reducing the grain size down to the ultrafine regime $(<1 \mu \mathrm{m})$ and thereby incorporating significant grain boundary (GB) strengthening [1, 2]. The ultrafine-grained (UFG) structure is expected to induce an influence on the precipitation behavior, because of the significantly reduced length scale. Nevertheless, there have been very limited studies on the effect of length scale on precipitation [3]. The present study was undertaken to obtain quantitative information on the differences in precipitation behavior between UFG structure and the traditional coarse-grained (CG) counterpart, and to provide fundamental insights into the underlying mechanisms. Al 7075 alloy, with major alloying elements of $\mathrm{Zn}, \mathrm{Mg}$ and $\mathrm{Cu}$, was selected for study because of its technological importance and the existing extensive studies on precipitation phenomena in CG Al 7075.

An UFG Al 7075 alloy was fabricated via powder cryomilling, degassing, hot isostatic pressing and extrusion, followed by solution treatment and artificial aging (T6 temper); a CG counterpart was prepared from unmilled CG powders using the same degassing, consolidation and heat treatment route [3]. The precipitation behavior in the UFG alloy was studied by atom-probe tomography (APT) and compared to that in its CG counterpart. APT studies were performed using a Cameca local-electrode atom-probe (LEAP) 4000X-Si tomograph with UV laser pulsing (355 nm wavelength), a pulse repetition rate of $500 \mathrm{kHz}$, and a pulse energy of $10-15 \mathrm{pJ}$.

Figure 1 (a) and (b) show the precipitates in the UFG and CG alloys, respectively, delineated by 8 at.\% $\mathrm{Zn}$ isoconcentration surfaces. Nanoscale Guinier-Preston (GP) zones (MgZn) and $\eta^{\prime}\left(\mathrm{MgZn}_{2}\right)$ precipitates were found in both alloys, as indicated by proximity histogram concentration profiles not displayed here. The majority of the precipitates is $\eta$ ' in the UFG alloy, whereas approximately half (number fraction) of the precipitates is $\eta$ ' in the CG counterpart. It is established that the precipitation in Al 7075 starts with the formation of Guinier-Preston (GP) zones, followed by the transformation of GP zones into $\eta$ ' [3]. The higher percent of $\eta$ '-precipitates in the UFG material indicates that the precipitation kinetics are enhanced by shorter diffusion distances and faster diffusion pathways at the GBs. The volume fraction of both types of precipitates in the UFG alloy is $\sim 3.7 \%$, and that in the CG counterpart is $\sim 5.4 \%$; the number density of precipitates in the former is $\sim 9.3 \times 10^{22} \mathrm{~m}^{-3}$, and that in the latter is $\sim 5.03 \times 10^{23} \mathrm{~m}^{-3}$. A significant number fraction of the precipitates was observed at GBs in the UFG alloy, whereas precipitates were predominantly found in grain interiors in the CG alloy. Figure 1(c) displays 2 at.\% $\mathrm{Cu}$ and 8 at.\% $\mathrm{Zn}$ isoconcentration surfaces in the UFG alloy. Cu segregation was found at GBs, which decorates the GBs and makes them easier to resolve. The significantly lower volume fraction and number density of precipitates in grain interiors in the UFG alloy relative to those in its CG counterpart are attributed to a smaller vacancy concentration in the UFG material. It has been 
demonstrated that the vacancy concentration is critically important for homogeneous nucleation of precipitates in Al-Zn-Mg-based alloys [3], whereas GBs act as sinks to absorb vacancies and cause annihilation. Since the UFG material possesses a significantly higher GB volume fraction than its CG counterpart, the vacancy concentration is expected to be distinctly smaller. Hence, homogenous nucleation will be hindered in grain interiors of UFG alloy. Nevertheless, precipitates can heterogeneously nucleate at GBs. Thus, a significant fraction of the precipitates in the UFG alloy is at GBs. The lower overall volume fraction and number density of precipitates in the UFG material relative to those in the CG counterpart indicate that the influence of impeded homogenous nucleation outweighs that of facilitated heterogeneous nucleation at GBs. The average precipitate diameter in the UFG alloy is similar to that in the CG counterpart $(\sim 5.0 \mathrm{~nm})$, whereas the former has significantly broader distribution of precipitate diameters, with some of the precipitates at GBs having distinctly larger diameters. The larger precipitate diameters at GBs can be ascribed to faster diffusion at GBs, which promotes precipitate growth. In addition, $\mathrm{MgO}$ dispersoids are evident in the UFG alloy, located at GBs and in grain interiors, which may serve as nucleation sites for precipitates. The volume fraction, number density and average diameter of $\mathrm{MgO}$ dispersoids are $\sim 0.4 \%, \sim 4.5 \times 10^{22} \mathrm{~m}^{-3}$ and $\sim 3.7 \mathrm{~nm}$, respectively.

In conclusion, homogeneous nucleation of precipitates in UFG Al 7075 is significantly inhibited, whereas heterogeneous nucleation is distinctly enhanced; the influence of the former outweighs that of the latter, leading to an overall smaller volume fraction and number density of precipitates in the UFG material relative to those in its $\mathrm{CG}$ counterpart. The differences in the precipitation behavior between UFG and CG alloys is rationalized on the basis of the influence of length scale on GB volume fraction, vacancy concentration in grain interiors, and diffusion kinetics and pathways.

\section{References:}

[1] YH Zhao et al., Acta Mater. 52 (2004), p. 4589.

[2] YH Zhao et al., Adv. Mater. 18 (2006), p. 2280.

[3] T Hu et al, Acta Mater. 61 (2013), p. 2163.

[4] Financial support from the Office of Naval Research (N00014-12-1-0237) is gratefully acknowledged. The LEAP tomograph at NUCAPT was purchased and upgraded with funding from NSF-MRI (DMR-0420532) and ONR-DURIP (N00014-0400798, N00014-0610539, N00014-0910781) grants. Instrumentation at NUCAPT was also supported by the Initiative for Sustainability and Energy at Northwestern (ISEN). This work was supported by the NSF's MRSEC program (DMR-0520513) and made use of its Shared Facilities at the Materials Research Center of Northwestern University (DMR1121262).

(a)

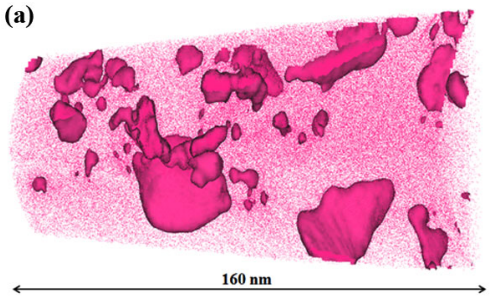

(b)

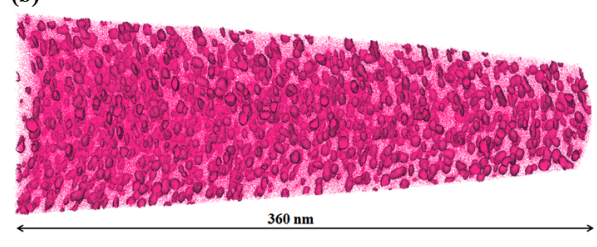

(c)

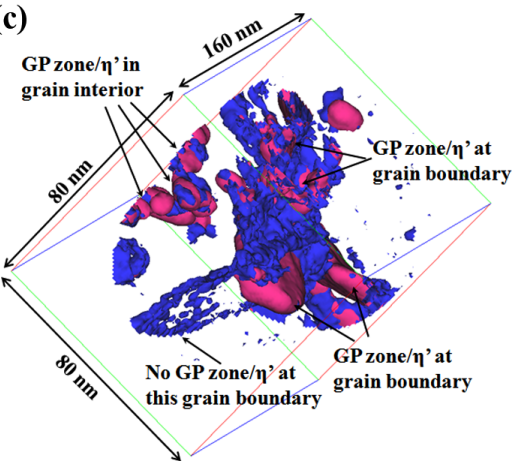

Figure 1. (a) (b) 8 at.\% $\mathrm{Zn}$ isoconcentration surface with $\mathrm{Zn}$ atoms superimposed for the UFG and CG Al 7075, respectively; (c) 2 at.\% $\mathrm{Cu}$ and 8 at.\% $\mathrm{Zn}$ isoconcentration surface for the UFG alloy. 